\title{
La transmission/reprise dans les PME: Une étude sur l'utilisation des ressources externes par les propriétaires dirigeants du Québec
}

\author{
Louise Cadieux, DBA \\ Université du Québec à Trois-Rivières \\ Martin Morin, professionnel de recherche \\ Université du Québec à Trois-Rivières \\ Institut de recherche sur les PME
}

Dans une perspective économique, assurer la continuité des PME par le biais d'une transmission (dans la perspective du prédécesseur/cédant) et/ou d'une reprise (dans la perspective du successeur/repreneur) présente des avantages non négligeables. Selon les rapports et études consultés sur le sujet, les repreneurs, qu'ils soient familiaux ou non, apportent, en effet, une nouvelle vigueur aux entreprises existantes procurant ainsi des retombées plus qu'intéressantes pour l'ensemble des parties prenantes ${ }^{1}$.

\section{Les repreneurs, qu'ils soient familiaux ou non, apportent, en effet, une nouvelle vigueur aux entreprises existantes}

Or, pour les prédécesseurs/cédants et pour les successeurs/repreneurs assurer la réussite de leurs projets est complexe. Pour les épauler dans leurs démarches respectives, plusieurs ressources humaines et financières sont d'ailleurs offertes sur le marché. À titre d'exemple, lors d'un mandat fait pour le compte du Ministère du Développement économique, de l'Innovation et de
l'Exportation du Québec (MDEIE), c'est au total 120 sites Web nationaux que nous avons retenus pour consultation, et ce plus précisément par les propriétaires dirigeants désireux d'assurer la transmission de leur entreprise. Donc, excluant les sites offrant de l'information ou des services aux nouveaux entrepreneurs espérant se partir en affaires par le biais d'une reprise.

Bien qu'il nous soit possible de connaître assez précisément l'offre de services en transmission ou en reprise d'entreprise, nous devons admettre ignorer les réelles habitudes d'utilisation de celles-ci, notamment chez les propriétaires dirigeants d'entreprises québécoises à qui le Ministère du Développement économique, de l'Innovation et de l'Exportation (MDEIE) désire venir en aide. Cet article a donc pour principal objectif de présenter les résultats d'une enquête faite auprès de propriétaires dirigeants ayant vécu au moins une expérience de transmission ou de reprise, plus précisément en ce qui concerne les ressources externes qu'ils ont utilisées pour les aider dans leurs démarches respectives. 


\section{L'utilisation des ressources externes par les propriétaires dirigeants de PME : une mise en contexte}

Selon certains, il est contre nature pour les propriétaires dirigeants de PME d'interagir

\author{
Dans la transmission/reprise \\ d'entreprise, le recours aux conseillers \\ externes doit être considéré
}

perspective des prédécesseurs/cédants, les résultats de l'enquête de la Fédération avec des conseillers externes, cela même s'ils $\mathrm{y}$ font régulièrement appel ${ }^{2}$. Les comptables agréés ne sont-ils pas parmi les conseillers les plus prisés par les propriétaires dirigeants de PME? Il semble, par ailleurs, que plusieurs types de conseillers externes aient aussi de la difficulté à établir un climat de collaboration avec les propriétaires dirigeants de PME, cela même lorsque ceux-ci requièrent leurs services de leur propre initiative. À ce propos, certains expliquent que le fossé entre les parties proviendrait, entre autres, du parcours académique des conseillers externes, lequel est principalement enrichi par des théories et concepts développés pour des grandes firmes et qui sont souvent malheureusement difficilement transférables dans d'autres contextes. Alors que d'autres l'expliquent par le manque d'arrimage entre les comportements entrepreneuriaux des propriétaires dirigeants de PME et ceux souvent plus programmés des conseillers externes ${ }^{3}$. L'inverse étant aussi envisageable. Pensons, dans le second cas, aux nombreux propriétaires dirigeants de PME peu enclins à la croissance ou favorisant des comportements stratégiques réactifs plutôt que ce que plusieurs considèrent comme proactifs ou, encore, entrepreneuriaux ${ }^{4}$.

Dans la transmission/reprise d'entreprise, le recours aux conseillers externes doit être considéré, tant pour les prédécesseurs/cédants que pour les successeurs/repreneurs. Du point de vue de la Commission Européenne (2006) ${ }^{5}$, de nombreux échecs auraient d'ailleurs pu être évités si, par exemple, les transmissions avaient été planifiées suffisamment à l'avance et l'avis d'experts demandé. Dans la
Canadienne des Entreprises Indépendantes ${ }^{6}$, montrent que ce sont $12 \%$ qui considèrent la non disponibilité de conseils professionnels comme un obstacle à la planification de la relève. Ce qui, de notre point de vue, indique un problème d'accès aux ressources externes mais aussi une bonne ouverture de leur part face à l'utilisation de celles offertes sur le marché. Chez les successeurs/repreneurs, le recours aux conseillers externes est aussi favorisé. Pensons, ici, à la préparation de leur projet d'affaires ou à la recherche de financement. Les résultats d'une enquête faite auprès de $725 \mathrm{PME}$ autrichiennes ayant été reprises entre 1996 et 2001 montrent, par exemple, que ce sont $80 \%$ des repreneurs qui ont eu recours à des conseillers externes de toutes catégories, dont principalement des experts en transfert de la propriété ${ }^{7}$.

S'intéresser aux ressources externes utilisées pour la transmission ou la reprise n'est pas nouveau. Certaines études ont d'ailleurs été faites auprès de ceux-ci, notamment en France. Parmi elles, nous retenons une enquête à laquelle ont participé $683 \mathrm{PME}$ françaises provenant de tous les secteurs d'activité révélant que, chez les prédécesseurs/cédants et les successeurs/repreneurs, ce sont des professionnels comme le comptable agréé, le banquier, le notaire et l'avocat qui sont priorisés pour les accompagner dans leur éventuelle opération de transmission et/ou de reprise $^{8}$. Toutefois, bien que ces études nous permettent de comprendre certains réflexes des propriétaires dirigeants à l'égard de l'utilisation des ressources externes en transmission ou en reprise, nous devons admettre ignorer de manière spécifique tant la 
nature des ressources utilisées par ceux-ci de même que les raisons pour lesquelles ils les utilisent, notamment chez une population de chefs d'entreprises québécoises pour qui il s'agit souvent de leur première expérience. Notre étude avait donc pour objectif spécifique de dresser un inventaire des

\section{La démarche de l'enquête}

Pour répondre aux objectifs de notre étude, nous avons effectué une enquête postale auprès de 1857 entreprises manufacturières et/ou de services québécoises préoccupées par la problématique de la transmission ou de la reprise. Pour les identifier, différentes stratégies ont été mises de l'avant, dont la collaboration avec les intervenants relève du Ministère du Développement économique, de l'Innovation et de l'Exportation (MDEIE) et la consultation de listes officielles provenant des régions et de la principale chercheure. Les questionnaires ont été postés le 11 janvier 2008, accompagnés d'une lettre de présentation invitant les propriétaires dirigeants à répondre pour le 28 janvier suivant. Vu le faible taux de réponse à la date de tombée, les questionnaires acceptés ont été considérés jusqu'au 4 mars 2008 alors que, pendant ce temps, une assistante de recherche faisait des rappels téléphoniques dans ressources externes utilisées tant par les prédécesseurs/cédants que par les successeurs / repreneurs québécois et de connaître leurs critères de choix ainsi que le degré de satisfaction à l'égard de l'utilisation de cellesci.
281 entreprises de trois régions, soit celles de la Mauricie, de la Montégérie et de Chaudières-Appalaches. Comme le montre le tableau 1, malgré les efforts déployés, des 195 questionnaires retournés par les propriétaires dirigeants sollicités (10,5\%), 49 ont été rejetés car insuffisamment complétés, de sorte que seuls 146 questionnaires valides ont pu être traités. Cela explique le taux de réponse final de 7,9\% lequel, en l'occurrence, est faible, notamment si l'on considère l'ampleur de la problématique et le nombre d'entreprises concernées par celle-ci. Enfin, même si l'étude n'était pas faite dans une perspective de comparaison, nous avons retenu que, parmi les 146 répondants, 92 étaient des prédécesseurs/cédants, pour une proportion de $63 \%$, tandis que 54 étaient des successeurs/repreneurs, pour une proportion de $37 \%$.

Tableau 1 Taux de réponse réparti selon les régions participantes

\begin{tabular}{|c|c|c|c|c|c|c|}
\hline & & \multicolumn{4}{|c|}{ Questionnaires } & \multirow{2}{*}{$\begin{array}{c}\text { Taux de réponse } \\
(\%)\end{array}$} \\
\hline No. & Région & postés & reçus & rejetés & valides & \\
\hline 1 & Bas St-laurent & 103 & 11 & 0 & 11 & 10,7 \\
\hline 2 & Saguenay Lac-St-Jean & 150 & 14 & 3 & 11 & 7,3 \\
\hline 3 & Capitale-Nationale & 140 & 17 & 4 & 13 & 9,3 \\
\hline 4 & Mauricie & 163 & 33 & 9 & 24 & 14,7 \\
\hline 5 & Estrie & 80 & 8 & 1 & 7 & 8,8 \\
\hline 6 & Montréal & 330 & 19 & 5 & 14 & 4,2 \\
\hline 7 & Outaouais & 32 & 1 & 0 & 1 & 3,1 \\
\hline 8 & Abitibi-Témiscamingue & 13 & 2 & 1 & 1 & 7,8 \\
\hline 9 & Côte-Nord & 100 & 8 & 2 & 6 & 6,0 \\
\hline 10 & Nord-du-Québec & 10 & 1 & 0 & 1 & 10,0 \\
\hline 11 & Gaspésie-Îles-de-la-Madeleine & 46 & 7 & 3 & 4 & 8,7 \\
\hline 12 & Chaudière-Appalaches & 136 & 17 & 5 & 12 & 8,8 \\
\hline 13 & Laval & 50 & 3 & 0 & 3 & 6,0 \\
\hline 14 & Lanaudière & 107 & 9 & 5 & 4 & 3,7 \\
\hline 15 & Laurentides & 149 & 5 & 2 & 3 & 2,0 \\
\hline 16 & Montérégie & 124 & 21 & 3 & 18 & 14,5 \\
\hline 17 & Centre-du-Québec & 124 & 11 & 2 & 9 & 7,3 \\
\hline \multicolumn{2}{|c|}{ Région non identifiée par les répondants } & & 8 & 4 & 4 & \\
\hline & Total & 1857 & 195 & 49 & 146 & 7,9 \\
\hline
\end{tabular}




\section{Les résultats de l'enquête}

Bien que la présente étude comporte plusieurs limites dont la principale provient du faible taux de réponse nous empêchant de généraliser les résultats à l'ensemble des PME québécoises préoccupées par la problématique de la transmission et de la reprise, ceux-ci permettent tout de même de faire certains constats originaux et éventuellement porteurs pour les parties prenantes. Mais avant d'en présenter les points saillants, voyons rapidement qui a répondu à notre enquête.

\subsection{Le profil des répondants}

D'abord, parmi l'ensemble des répondants, retenons que $91,7 \%$ sont des hommes; 49,3\% sont âgés de plus de 55 ans et que $41,4 \%$ ont un diplôme d'études de niveau universitaire. Toutefois, bien que cette enquête n'ait pas été faite pour des fins de comparaison, nous avons tout de même distingué les deux catégories de répondants, soit les prédécesseurs/cédants et les successeurs/repreneurs. La figure 1 illustre les principales distinctions entre ceux-ci, notamment au chapitre de l'âge, du sexe (il y aurait plus de femmes chez les successeurs /repreneurs) et du degré de scolarité (plus élevé chez les successeurs/repreneurs). De tous les répondants, 69,2\% étaient à la tête d'entreprises $\mathrm{du}$ secteur manufacturier et $29,5 \%$ du secteur des services; 39,2\% procuraient de l'emploi à moins de 20 personnes; $31,5 \%$ à entre 20 et 49 personnes; $29,4 \%$ à 50 personnes et plus. Notons aussi que $6,9 \%$ des entreprises répondantes généraient, au moment de l'enquête, un chiffre d'affaires annuel de moins de $500000 \$ ; 11 \%$ entre $500000 \$$ et
$1 \mathrm{M} \$ ; 47,6 \%$ entre $1 \mathrm{M} \$$ et $5 \mathrm{M} \$ ; 15,9 \%$ entre $5 \mathrm{M} \$$ à $10 \mathrm{M} \$$; et $18,6 \%$ faisaient plus de $10 \mathrm{M} \$$. Pour sa part, la figure 2 , résume les principales caractéristiques des entreprises participantes toujours selon les catégories de répondants. Sur ce point, nos résultats montrent qu'en matière de secteur d'activité et de taille, les entreprises ont pratiquement le même profil, quel que soit le type de répondants (prédécesseurs/cédants - successeurs / repreneurs).

\section{$\mathbf{7 4 , 8} \%$ des prédécesseurs/cédants ayant participé à notre enquête sont à la tête d'une entreprise qu'ils ont fondée ou acquise avant 1990}

Enfin, $74,8 \%$ des prédécesseurs/cédants ayant participé à notre enquête sont à la tête d'une entreprise qu'ils ont fondée ou acquise avant 1990. La moyenne étant 1986. Âgés en moyenne de 58 ans, ceux-ci sont en affaires depuis en moyenne 22 ans, dont près du tiers (28\% à $30 \%)$ prévoit quitter la direction et la propriété de leur entreprise entre 2008 et 2010. Chez les successeurs/repreneurs, le profil est différent. À la tête de leur entreprise depuis en moyenne 1998 et âgés, aussi en moyenne, de 46 ans, $81,5 \%$ ont répondu qu'il était trop tôt pour penser à la transmission de leur entreprise. Les deux profils étant passablement distincts à plusieurs égards, nos résultats montrent dans quelle mesure il peut être important de s'adresser aux deux catégories de propriétaires dirigeants lorsqu'il est question de comprendre la problématique nous concernant. 
Figure 1 Le profil des propriétaires dirigeants

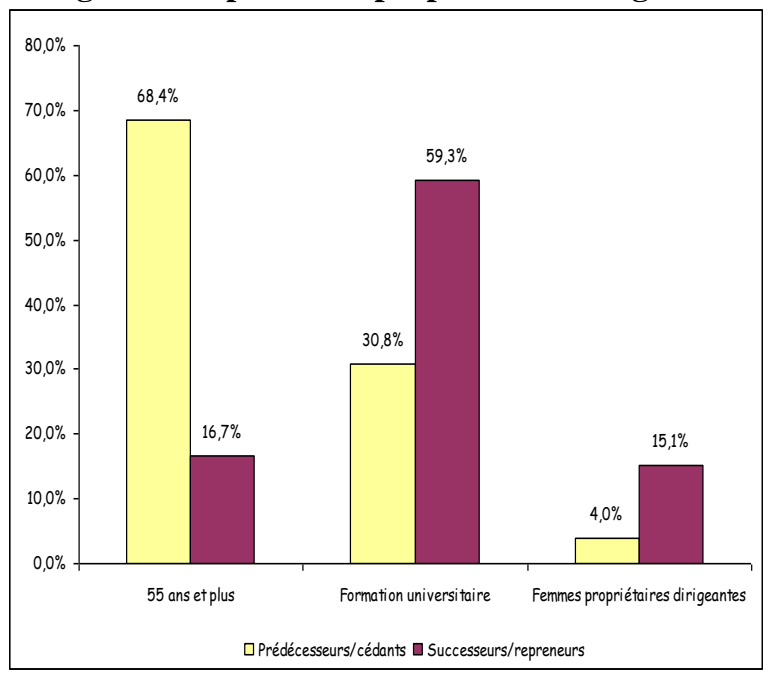

3.2 Les difficultés de la transmission et de la reprise

Malgré que la littérature professionnelle et académique existante nous permette de comprendre les difficultés de la transmission et de la reprise, peu se sont intéressés à les prioriser. Nous avons donc demandé aux répondants d'indiquer, sur une échelle de 5 points $(1=$ très facile et $5=$ très difficile), le degré perçu de difficulté pour chacun des énoncés soumis selon chacune des catégories de répondants, soit les prédécesseurs/cédants et les successeurs / repreneurs. À ces questions, le tableau 2 révèle
Figure 2 Profil des entreprises répondantes

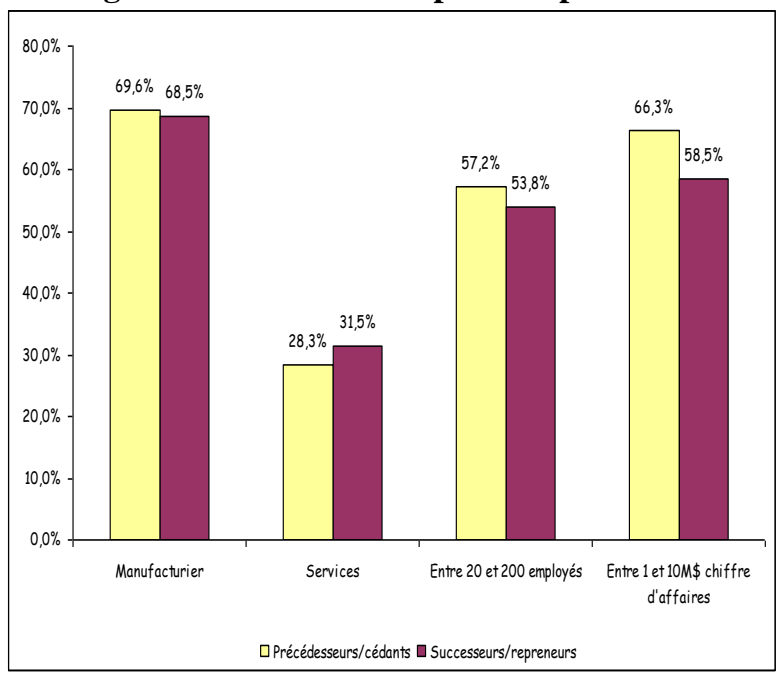

que des quatre premières difficultés perçues par les prédécesseurs / cédants, nous devons retenir, dans l'ordre, celles de préparer et de planifier leur relève $(3,38 / 5)$, de trouver des candidats à la relève sérieux et compétents $(3,34 / 5)$, de quitter définitivement la direction de l'entreprise $(3,28 / 5)$ et d'évaluer le juste prix de vente de l'entreprise (3,27/5). Difficultés qui, dans les trois premiers cas, concernent moins le transfert de la propriété et plus la capacité des prédécesseurs/cédants à se retirer de leur entreprise. Cette dimension de la problématique est d'ailleurs fortement documentée dans la littérature professionnelle et scientifique depuis bon nombre d'années ${ }^{9}$.

Tableau 2 Les difficultés perçues chez les prédécesseurs/cédants

\begin{tabular}{|c|c|c|}
\hline Énoncés des difficultés & $\begin{array}{c}\% \text { où niveau }=\text { difficile (4) } \\
\text { ou très difficile (5) }\end{array}$ & $\begin{array}{c}\text { moyenne } \\
15\end{array}$ \\
\hline Préparer et planifier ma relève & 43,7 & 3,38 \\
\hline Trouver des candidats à la relève sérieux et compétents & 44,1 & 3,34 \\
\hline Quitter définitivement la direction de mon entreprise & 41,2 & 3,28 \\
\hline Évaluer le juste prix de vente de mon entreprise & 48,5 & 3,27 \\
\hline Trouver des partenaires financiers pour mon projet de transmission & 35,3 & 3,00 \\
\hline Trouver du soutien en matière de relève & 29,0 & 2,90 \\
\hline Mobiliser le personnel clé avant, pendant et après la transmission & 25,7 & 2,89 \\
\hline Transmettre mes connaissances au $(x)$ candidat $(s)$ à la relève & 30,4 & 2,86 \\
\hline Assurer une bonne transition avec le candidat à la relève & 20,0 & 2,67 \\
\hline Transmettre mon capital relationnel (réseau d'affaires) & 23,2 & 2,64 \\
\hline Intéresser mes enfants à l'entreprise & 20,3 & 2,44 \\
\hline Parler ouvertement de la transmission de mon entreprise & 17,1 & 2,40 \\
\hline Fournir des informations confidentielles aux candidats à la relève & 19,4 & 2,27 \\
\hline Mobiliser les parties prenantes (fournisseurs, clients, etc,) & 4,4 & 2,26 \\
\hline Parler de la transmission de mon entreprise avec les membres de ma famille & 12,9 & 2,16 \\
\hline Total & 27,7 & 2,80 \\
\hline
\end{tabular}


Quant aux successeurs/repreneurs, le tableau 3 révèle que les quatre dimensions perçues les plus difficiles pour eux sont, en ordre d'importance, négocier le prix et les conditions d'achat de l'entreprise (2,97/5), trouver une ou des entreprises à reprendre $(2,82 / 5)$, trouver du soutien en matière de reprise $(2,74 / 5)$ et trouver et obtenir du financement $(2,65 / 5)$ ou trouver des partenaires financiers $(2,63 / 5)$. Représentant, dans leur cas, de plus grandes difficultés ressenties au chapitre du transfert de la propriété. À ces difficultés, s'ajoute aussi celle concernant la présence du prédécesseur/cédant dans l'entreprise où $38,9 \%$ des répondants estiment cela difficile ou très difficile. Nos résultats sont donc représentatifs de ce que nous comprenons déjà de cette dimension de la problématique de la transmission/reprise, notamment à l'aide des études faites en France sur ce sujet ${ }^{10}$.

Cette compréhension spécifique des difficultés perçues selon la catégorie de répondants pourrait nous permettre de croire que ceux-ci utilisent des ressources externes de différentes natures. Par exemple, nous pourrions supposer que les successeurs/repreneurs ont plus tendance à chercher conseil auprès d'experts en transfert de la propriété, ce qui est leur première préoccupation et moins auprès de ceux spécialisés en transfert de la direction. Au même titre que nous pourrions supposer que les prédécesseurs/cédants sont plus susceptibles d'utiliser des ressources externes expertes en transfert de la direction, vu leurs difficultés ressenties face à leur éventuel retrait des affaires courantes de leur entreprise. Mais, voyons, ci-après, ce que nos participants ont répondu à cet effet.

\section{Nos résultats sont donc représentatifs de ce que nous comprenons déjà de cette dimension de la problématique de la transmission/reprise}

Tableau 3 Difficultés perçues chez les successeurs/repreneurs

\begin{tabular}{|c|c|c|}
\hline Énoncés des difficultés & $\begin{array}{c}\% \text { où niveau }=\text { difficile (4) ou } \\
\text { très difficile (5) }\end{array}$ & $\begin{array}{c}\text { moyenne } \\
15\end{array}$ \\
\hline Négocier le prix et les conditions d'achat de l'entreprise & 38,9 & 2,97 \\
\hline Trouver une ou des entreprises à reprendre & 33,3 & 2,82 \\
\hline Trouver du soutien en matière de reprise d'entreprise & 25,7 & 2,74 \\
\hline Trouver et obtenir du financement & 27,0 & 2,65 \\
\hline La présence du prédécesseur/cédant dans l'entreprise & 38,9 & 2,64 \\
\hline Trouver des partenaires financiers pour mon projet de reprise & 22,9 & 2,63 \\
\hline Avoir toute l'information sur l'entreprise à reprendre & 27,8 & 2,42 \\
\hline Faire affaires avec le(s) professionnel(s) ou conseiller $(s)$ du prédécesseur/cédant & 22,2 & 2,42 \\
\hline Parler ouvertement de mon projet de reprise & 13,9 & 2,31 \\
\hline Assurer une bonne transition avec le prédécesseur/cédant & 13,9 & 2,31 \\
\hline Intégrer le réseau d'affaires du prédécesseur/cédant & 16,7 & 2,25 \\
\hline Développer mon propre réseau d'affaires & 13,9 & 2,22 \\
\hline Se faire accepter des employés & 13,9 & 2,06 \\
\hline Parler de mon projet de reprise avec les membres de ma famille & 8,1 & 2,00 \\
\hline Total & 22,7 & 2,46 \\
\hline
\end{tabular}

\subsection{Les ressources externes utilisées}

Parmi les objectifs de l'enquête, nous voulions dresser la liste des ressources externes utilisées par les propriétaires dirigeants préoccupés par la transmission ou la reprise d'une entreprise. Nous avons donc soumis une liste exhaustive des conseillers ou des experts pouvant intervenir dans la démarche et demandé aux répondants d'identifier ceux auprès de qui ils avaient fait affaires avant, pendant et après le processus de la transmission ou de la reprise. À ce sujet, et comme l'illustre la figure 3 , nos résultats 
indiquent que pour l'ensemble des répondants, ce sont, en ordre d'importance : le comptable agréé $(95,1 \%)$, le fiscaliste $(79,9 \%)$, le notaire $(65,3 \%)$, le banquier $(59,0 \%)$, l'avocat $(44,4 \%)$, le courtier en assurances $(35,4 \%)$, le planificateur financier $(29,9 \%)$ qui sont les plus souvent impliqués dans les dossiers de transmission et ou de reprise. Viennent ensuite, pour moins du quart des répondants, le coach ou le mentor $(22,9 \%)$, le conseiller en management $(14,6 \%)$, le conseiller en gestion des ressources humaines $(9,7 \%)$, le conseiller gouvernemental $(10,4 \%)$, le conseiller en développement organisationnel $(6,9 \%)$, le psychologue industriel $(6,3 \%)$, le psychologue personnel $(2,1 \%)$ et le psychologue de la famille $(2,1 \%)$.

Figure 3 Les ressources externes utilisées

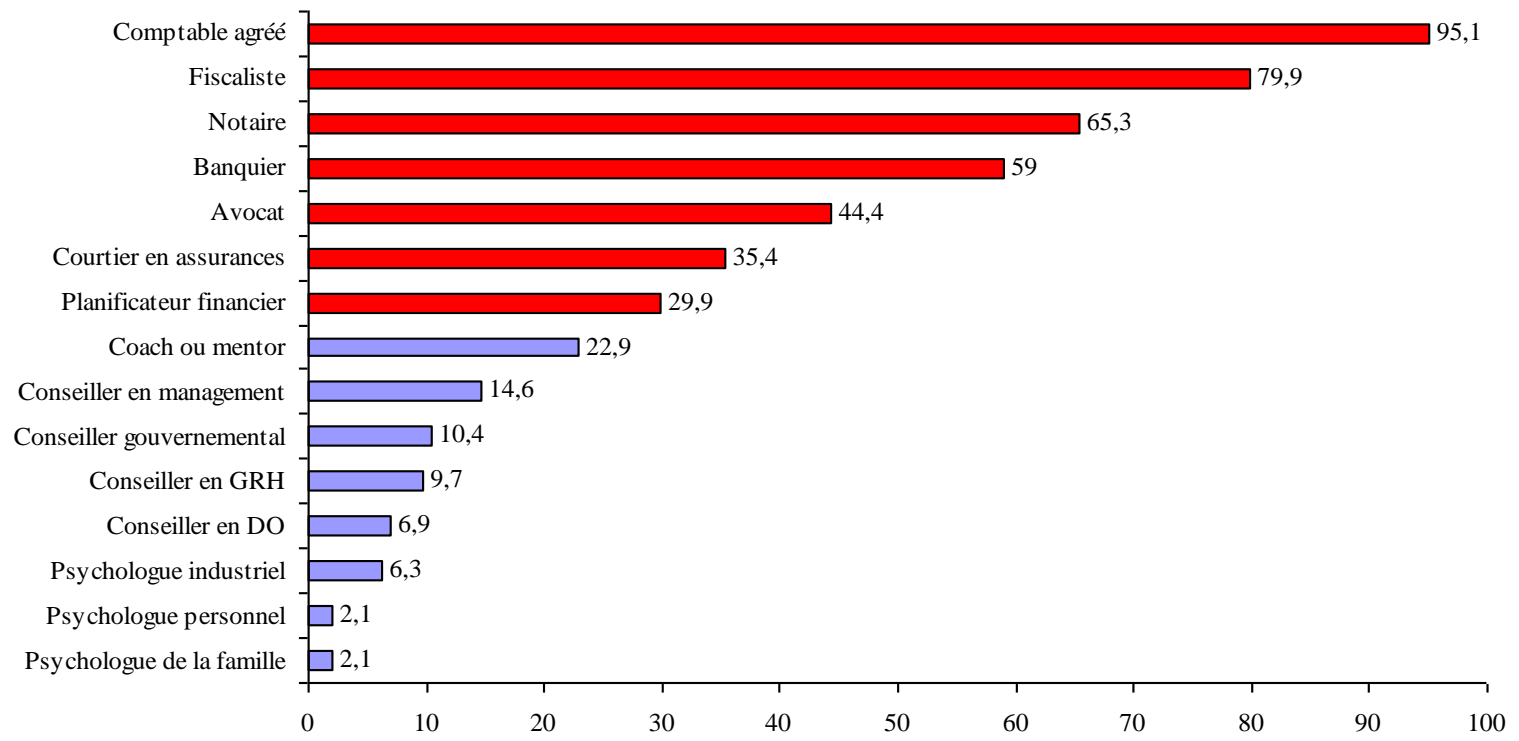

Pour assurer la transmission ou la reprise d'une entreprise, deux transferts sont nécessaires, soit celui de la direction et celui de la propriété. Sur ce point, nous aimerions rappeler que le transfert de la propriété concerne plus spécifiquement les aspects légaux, dont la finalité est la possession du capital actions ou des actifs de l'entreprise par les successeurs/repreneurs. Dans cette perspective, il sera question d'assurer ce transfert ou de sécuriser le fond de retraite des prédécesseurs/cédants. Pour sa part, le transfert de la direction s'intéresse au changement du leadership. Pour l'achever, il faut par exemple, reconnaître les candidats à la relève sérieux et les préparer en conséquence. Les problématiques y étant liées sont donc souvent considérées dans une perspective plus «humaine » ou «managériale ». Ce qui se distingue par des besoins plus spécifiques en matière d'utilisation des ressources externes. Pensons, par exemple, à l'évaluation des compétences du candidat à la relève, à son plan de formation et de préparation, à la capacité du prédécesseur/ cédant à laisser sa place ou à l'ouverture des membres de la famille ou de l'entreprise à accepter un nouveau dirigeant ${ }^{11}$. 
C'est dans cette perspective que nous avons voulu connaître de façon détaillée les différentes ressources externes utilisées par les propriétaires dirigeants en matière de transmission pour chacun des types de transfert associés à la transmission. D'abord, comme le montre le tableau 4, en réponse à cette dimension de la question, il ressort avant tout que les experts en transfert de propriété sont plus utilisés. Qui plus est, ils sont aussi très sollicités même pour des aspects qui concernent le transfert de la direction, et ce, encore plus que des experts spécialisés à cette fin, tels les psychologues industriels ou les conseillers en management. Du reste, pour l'ensemble des répondants, nos résultats montrent que c'est la majorité qui a consulté un comptable agréé, tant en ce qui concerne les aspects liés au transfert de la direction $(67,4 \%)$ que celui de la propriété $(84,0 \%)$. Ensuite, vient le fiscaliste, dans une proportion de $43,1 \%$ pour le transfert de la direction et $70,1 \%$ pour le transfert de la propriété. Notons, au passage, que cela est semblable pour les deux catégories de répondants, à savoir les prédécesseurs/cédants et les successeurs/repreneurs.

Tableau 4 Les ressources externes utilisées par type de transfert

\begin{tabular}{|c|c|c|c|c|c|c|}
\hline \multirow{2}{*}{$\begin{array}{c}\text { \% de répondants qui ont utilisé cette } \\
\text { ressource }\end{array}$} & \multicolumn{2}{|c|}{$\begin{array}{l}\text { Prédécesseurs/ } \\
\text { cédants }\end{array}$} & \multicolumn{2}{|c|}{$\begin{array}{l}\text { Successeurs/ } \\
\text { repreneurs }\end{array}$} & \multicolumn{2}{|c|}{ Total } \\
\hline & direction & propriété & direction & propriété & direction & propriété \\
\hline \multicolumn{7}{|c|}{ Experts en transfert de propriété } \\
\hline Le comptable agréé & 68,9 & 81,1 & 64,8 & 88,9 & 67,4 & 84,0 \\
\hline Le fiscaliste & 47,8 & 70,0 & 35,2 & 70,4 & 43,1 & 70,1 \\
\hline Le banquier & 36,7 & 46,7 & 38,9 & 57,4 & 37,5 & 50,7 \\
\hline Le notaire & 34,4 & 50,0 & 42,6 & 68,5 & 37,5 & 56,9 \\
\hline Le planificateur financier & 20,0 & 32,2 & 11,1 & 14,8 & 16,7 & 25,7 \\
\hline L'avocat & 26,7 & 35,6 & 16,7 & 42,6 & 22,9 & 38,2 \\
\hline Le courtier en assurances & 20,0 & 25,6 & 20,4 & 37,0 & 20,1 & 29,9 \\
\hline $\begin{array}{l}\text { Le conseiller de mon institution } \\
\text { financière }\end{array}$ & 15,6 & 18,9 & 16,7 & 22,2 & 16,0 & 20,1 \\
\hline \multicolumn{7}{|c|}{ Experts en transfert de direction } \\
\hline Le psychologue industriel & 6,7 & 1,1 & 3,7 & 3,7 & 5,6 & 2,1 \\
\hline Le psychologue de la famille & 1,1 & 1,1 & 3,7 & 1,9 & 2,1 & 1,4 \\
\hline Le psychologue personnel & 1,1 & 0,0 & 3,7 & 1,9 & 2,1 & 0,7 \\
\hline Le conseiller en management & 16,7 & 11,1 & 7,4 & 7,4 & 13,2 & 9,7 \\
\hline Le conseiller gouvernemental & 6,7 & 5,6 & 7,4 & 13,0 & 6,9 & 8,3 \\
\hline $\begin{array}{l}\text { Le conseiller en gestion des } \\
\text { ressources humaines }\end{array}$ & 10,0 & 5,6 & 5,6 & 3,7 & 8,3 & 4,9 \\
\hline $\begin{array}{l}\text { Le conseiller en développement ou en } \\
\text { changement organisationnel }\end{array}$ & 7,8 & 3,3 & 5,6 & 1,9 & 6,9 & 2,8 \\
\hline Le coach ou le mentor personnel & 15,6 & 12,2 & 24,1 & 22,2 & 18,8 & 16,0 \\
\hline Autre(s) & 0,0 & 0,0 & 5,6 & 5,6 & 2,1 & 2,1 \\
\hline Total & 19,8 & 23,5 & 18,4 & 27,2 & 19,2 & 24,9 \\
\hline
\end{tabular}

Ensuite, viennent, toujours en ordre d'importance, le banquier, le notaire, le planificateur financier, l'avocat, le courtier en assurances et le conseiller de l'institution financière. Les ré-

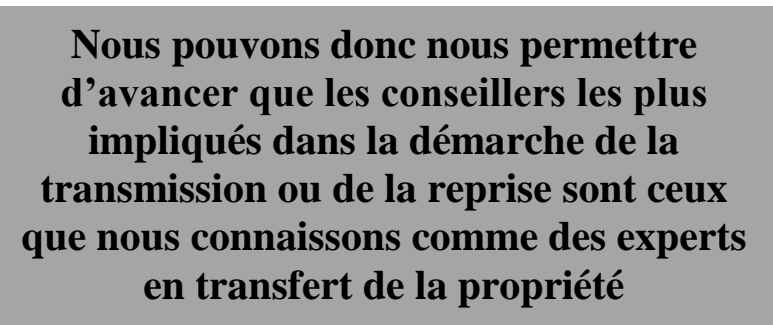

de conseillers, comme les psychologues industriels, les psychologues personnels ou les psychologues de la famille ont été les moins sollicités par nos répondants. sultats montrent aussi dans quelle mesure peu recourent à des experts en transfert de la direction. À leur sujet, il appert que ce sont le coach ou le mentor qui sont les plus souvent interpellés pour une proportion de $16 \%$, tandis que les autres types
À la lumière de nos résultats, nous pouvons donc nous permettre d'avancer que les conseillers les plus impliqués dans la démarche de la transmission ou de la reprise sont ceux que nous connaissons comme des experts en transfert de la 
propriété. Toutefois, une compilation différente des résultats laisse croire qu'il existerait, malgré tout, une certaine cohérence dans le comportement des participants vis-à-vis les ressources externes utilisées en fonction du type de transfert (propriété vs direction). Nos résultats, résumés au tableau 5 , révèlent, en effet, que lorsque ceux-ci font appel à des conseillers, ils les utilisent plus souvent pour leurs compétences respectives. Cela s'explique, d'abord, par le fait que $34,7 \%$ des répondants ont eu recours à un ou des conseillers en transfert de direction pour les aider dans le transfert de la direction, comparé à 29,2\% qui ont utilisé ces mêmes conseillers pour le transfert de la propriété. Et, ensuite, par le constat que 90,3\% d'entre eux ont eu recours à au moins un conseiller en transfert de la propriété, mais que ce sont $77,1 \%$ de ces mêmes répondants qui les ont utilisés pour les aider dans le transfert de la direction.

Tableau 5 Les types de ressources externes utilisées selon le type de transfert

\begin{tabular}{|l|c|c|}
\hline \multirow{2}{*}{$\%$ de répondants qui ont utilisé au moins une ressource } & \multicolumn{2}{|c|}{ Type de transfert } \\
\cline { 2 - 3 } & direction & propriété \\
\hline Un ou des conseillers en transfert de propriété & 77,1 & 90,3 \\
\hline Un ou des conseillers en transfert de direction & 34,7 & 29,2 \\
\hline
\end{tabular}

Nous gardons toutefois en mémoire la constance de la forte tendance chez les proprié-

\section{Les revues et les journaux d'affaires sont la deuxième source d'information la plus utilisée}

externes peuvent être considérées. Il peut, par exemple, s'agir des collèges et des univertaires dirigeants de PME québécoises pour l'utilisation des services de conseillers spécialisés en transfert de la propriété à la faveur des conseillers en transfert de la direction. Ce qui, sans surprise, corrobore les résultats d'autres enquêtes comme celle de la FCEI (2005), et que nous expliquons, du coup, par le lien de confiance existant entre le propriétaire dirigeant et le conseiller externe, surtout lorsqu'il s'agit de discuter de sujets personnels comme ce qu'il advient du patrimoine familial, du partage de celui-ci ou plus simplement de ses propres besoins financiers une fois retiré de ses activités professionnelles. Nous nous permettons, ici, de rappeler que ces points sont parmi les principales préoccupations (mais pas des difficultés perçues) des propriétaires dirigeants lorsqu'il est question de la transmission de leur entreprise.

\subsection{Les autres ressources externes utilisées par les répondants}

En matière de transmission et de reprise d'entreprise, d'autres formes de ressources sités, des organismes publics et/ou des associations d'affaires auxquelles les protagonistes appartiennent et qui offrent des services ou des produits en relève d'entreprise. De notre point de vue, tous ces organismes, qu'ils soient privés, publics ou parapublics, ont un travail d'accompagnement et de formation à effectuer auprès des propriétaires dirigeants soucieux d'assurer la transmission de leur entreprise et des candidats à la relève désireux de reprendre une entreprise déjà bien établie. À ce propos, les résultats de notre étude, illustrés à la figure 4, montrent que 52,7\% d'entre eux utilisent leurs réseaux de contacts comme autres ressources externes. Les revues et les journaux d'affaires sont la deuxième source d'information la plus utilisée, pour une proportion de 39,0\% des répondants. Tandis que les associations d'affaires arrivent bonnes troisièmes, pour une proportion de 32,9\%. Ensuite viennent les colloques sur la relève, pour $27,4 \%$ et les documents provenant des divers gouvernements pour $19,2 \%$.

Nos résultats montrent aussi que seuls 15,8\% de l'ensemble des répondants participe à des 
ateliers de sensibilisation sur la relève. Alors qu'il s'agit d'une stratégie répandue et grandement utilisée depuis les dernières années. La très faible proportion d'entre eux qui s'inscrivent à des formations sur mesure $(9,6 \%)$ ou offertes dans les universités $(4,1 \%)$ et les cégeps $(0,7 \%)$ est aussi surprenante puisque, là aussi, plusieurs formations sont offertes sur une base régulière, et ce, un peu partout à travers le Québec. Enfin, nous avons remarqué que, pour les «autres» ressources externes utilisées dont nous venons de parler, ce sont les prédécesseurs/cédants qui les utilisent le plus souvent, dans une proportion de $22,4 \%$ comparé à $13,9 \%$ chez les successeurs / repreneurs. Ce qui laisse supposer que le propriétaire dirigeant préoccupé par la continuité de son entreprise est plus enclin à chercher de l'information variée sur la problématique que le candidat à la relève soucieux de se partir en affaires, par le biais d'une reprise.

Figure 4 Les autres ressources utilisées par les propriétaires dirigeants de PME

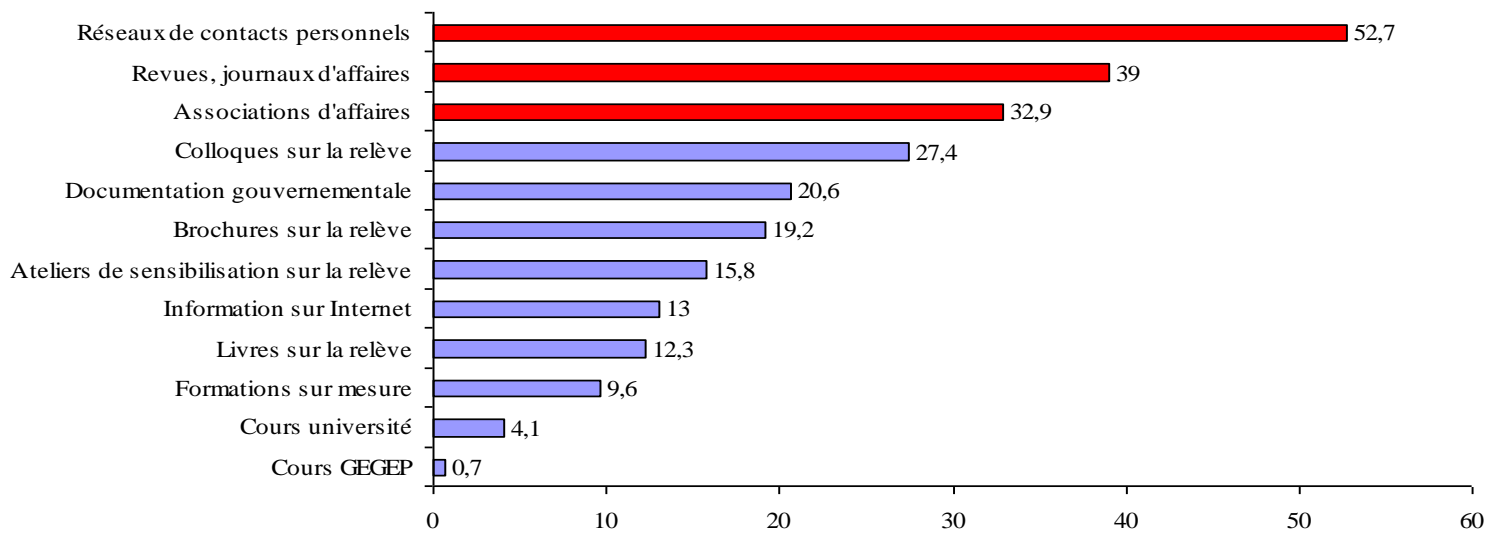

3.5 Les critères de choix et la satisfaction à l'égard des ressources externes utilisées

Dans cette étude, nous avons voulu connaître les critères utilisés par les propriétaires dirigeants dans le choix des ressources externes qu'ils utilisent. Comme le révèle la figure 5 , parmi les résultats, nous retenons que $84,9 \%$ d'entre eux choisissent un conseiller externe parce qu'ils font déjà affaire avec lui. Alors que $54,8 \%$ font affaire avec un conseiller parce qu'ils le connaissent déjà, sans nécessairement faire affaire avec lui (les répondants pouvaient cocher plusieurs énoncés). Ce qui explique, en partie, que ce soit le comptable agréé qui soit parmi les conseillers les plus sollicités, étant celui qui est le plus susceptible d'être auprès du propriétaire dirigeant depuis qu'il est en affaires. D'autres motivations sont toutefois exprimées par un peu moins du quart des répondants. Parmi celles-ci, nous retenons la référence d'un membre du réseau d'affaires du propriétaire dirigeant, dans $24,7 \%$ des cas et le fait que ce dernier le connaissait de réputation dans $15,8 \%$ des cas. Les résultats de cette étude nous permettent donc de croire que les propriétaires dirigeants de PME font d'abord affaire avec des conseillers externes provenant de leur entourage immédiat et avec qui ils ont développé un lien de confiance et, ensuite, avec ceux dont ils ont déjà entendu parler ou qui sont référés par des personnes de leur entourage immédiat. 
Figure 5 Motivations du choix des ressources externes utilisées

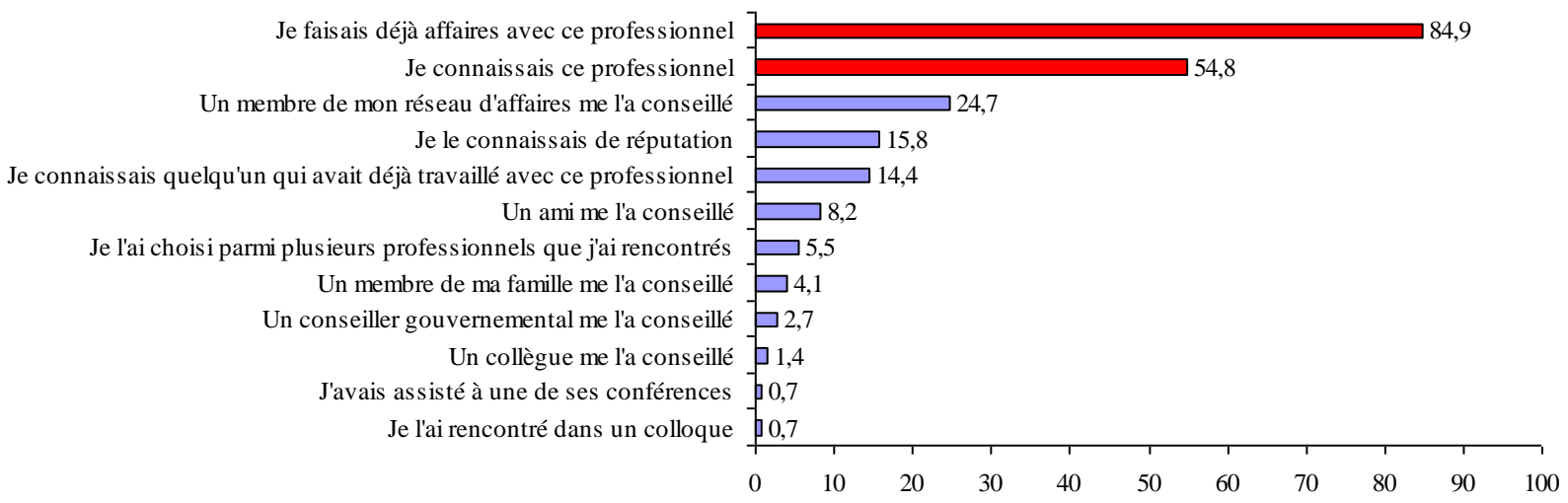

Or à ce sujet, nos résultats montrent une certaine incohérence dans l'utilisation des ressources externes chez les répondants puisque la majorité dit trouver «plutôt difficile », en ordre d'importance, de connaître les compétences à rechercher chez les professionnels devant les accompagner dans leur démarche $(3,21 / 5)$, de connaître les bons professionnels en matière de transmission $(3,09 / 5)$ et d'avoir toute l'information disponible sur les ressources disponibles $(3,03 / 5)$. Viennent ensuite des difficultés comme avoir de l'information pertinente au sujet des professionnels pouvant les conseiller $(2,99 / 5)$ ou trouver des personnes de confiance et compétentes pour les conseiller $(2,91 / 5)$, laissant ainsi supposer que les propriétaires dirigeants, préoccupés par la transmission ou la reprise d'une entreprise, trouvent principalement autour d'eux les ressources externes dont ils ont besoin mais sans nécessairement savoir s'il s'agit des bonnes.

\section{Conclusion}

Cette enquête avait pour objectif de connaître les habitudes d'utilisation des ressources externes chez les propriétaires dirigeants des PME québécoises vivant ou ayant vécu une expérience de transmission ou de reprise. Comme nous l'avons vu dans les pages précédentes, malgré un faible taux de réponse, les résultats présentés favorisent tout de même
Sur ce dernier point, notons que les participants à notre enquête se disaient «satisfaits» des ressources externes qu'ils utilisent (pour une moyenne totale de 3,89/5), et ce, assez pour les recommander fortement à leur entourage (91,6\% en moyenne). D'autant plus que, de leur point de vue, les inconvénients sont moins importants que les avantages retirés des ressources externes qu'ils utilisent. Ce qui indique, encore une fois, combien le lien de confiance existant entre les propriétaires dirigeants et les conseillers est considéré plus important que les compétences à rechercher chez ces derniers. Plus simplement, à partir du moment où ils se sentent en confiance, les propriétaires dirigeants de PME sont susceptibles de porter un jugement positif sur les compétences des personnes auprès de qui ils prennent conseil, cela même s'ils admettent ne pas connaître les compétences à rechercher chez ceux-ci, notamment lorsque la continuité de l'entreprise est le principal enjeu.

quelques pistes de réflexion intéressantes. Par exemple, nos résultats montrent dans quelle mesure la transmission est une problématique comportant une dimension de nature personnelle pour les propriétaires dirigeants des PME québécoises. Ce constat s'explique, entre autres, par les «patterns » qu'ils utilisent pour choisir les conseillers externes avec qui 
ils font affaire au cours du processus. Nos résultats montrent, en effet, que pour sélectionner leurs conseillers externes en transmission, ils favorisent ceux avec qui ils font déjà affaire ou qu'ils connaissent ou qui sont référés par leurs réseaux. Priorisant ainsi le lien de confiance ou la réputation de ceuxci, alors qu'ils admettent ne pas connaitre toutes les compétences à rechercher chez ceux-ci. Nos résultats laissent donc supposer que, lorsqu'il est question de choisir des ressources externes, les propriétaires dirigeants de PME québécoises accordent plus d'importance à des facteurs subjectifs au dépend d'autres facteurs plus objectifs, pourtant considérés importants dans la littérature académique et professionnelle ${ }^{12}$. Pensons, par exemple à la formation ou à l'expérience du conseiller externe en transmission ou à tout autre compétence liée à son domaine d'expertise, comme la capacité de donner des conseils sur la manière dont le propriétaire dirigeant doit préparer ses employés aux changements qui surviendront dès le moment où le candidat à la relève est choisi.

Du reste, nous retenons dans quelle mesure l'ensemble des propriétaires dirigeants de PME québécoises fait appel à des experts en transfert de la propriété, cela malgré la nature des difficultés perçues de nature plus personnelle ou managériale, comme se retirer de leur entreprise ou trouver des candidats à la relève compétents et intéressés. D'autant plus que, parmi les questionnaires rejetés, plusieurs répondants disaient ne pas être rendus à la phase de consultation d'experts, alors qu'ils avaient répondu avoir une relève identifiée, en formation ou en place dans leur entreprise. $\mathrm{Ce}$ qui, de notre point de vue, indique une compréhension limitée de la problématique de la transmission ou de la reprise d'entreprise par les propriétaires dirigeants de PME québécoises. La note positive est que ce constat laisse encore de la place pour des activités de sensibilisation, autres que les formations sur mesure, les colloques ou les ateliers sur la relève qui ne semblent intéresser qu'une faible proportion de la population cible. La dimension très personnelle de la problématique de la transmission explique, en retour, le faible taux de participation des propriétaires dirigeants à des activités de sensibilisation ou à des formations au cours desquelles ils se sentiraient dans l'obligation de partager leurs préoccupations avec des personnes qu'ils ne connaissent pratiquement pas ou pas du tout. Le problème du peu de participation découle donc d'un phénomène plus important que celui du manque de temps souvent invoqué de leur part lorsqu'ils sont questionnés à ce sujet. Bref, les intervenants gouvernementaux de même que les parties prenantes ont encore beaucoup de travail à faire pour aider les PME à réussir leurs projets de continuité. Bien sûr, en autant qu'ils se permettent de libérer leur potentiel de créativité, ce qu'ils sont tout à fait capables de faire.

\section{Notes et références}

1 Voir à ce sujet Counot, S. et Mulic, S. (2004). Le rôle économique des repreneurs d'entreprise. INSEE Première, no. 975, www.ceder-reprendre-une-entreprise.com; Commission Européenne (2006). Communication de la commission au conseil, au parlement européen, au comité économique et social et au comité des régions. Bruxelles, 14 mars. www.ceder-reprendre-uneentreprise.com; OSEO (2005). La transmission des petites et moyennes entreprises : l'expérience d'OSEO bdpme. http://www.oseo.fr/IMG/pdf/Oseo_transmission.pdf

2 Voir à ce sujet Christensen, P.-R. et Klyver, K. (2006). Management consultancy in small firms: how does interaction work, Journal of Small Business and Enterprise Development, 13(3), 299-313; Raymond, L. Blili, S, et El Alami, D. (2004). L'écart entre le consultant et la PME : analyse et perspectives, Revue internationale de gestion, 28(4), 52-60; Ribiero Soriano, D. (2003). The impact of consulting service on Spanish Firms, Journal of Small Business Management, 41(4a), 409-415.

3 Voir Raymond, L. Blili, S, et El Alami, D. (2004). L'écart entre le consultant et le PME : analyse et perspectives, Revue internationale de gestion, 28(4), 52-60; et Day, J., Reynolds, P. et Lancaster, G. (2006). Entrepreneurship and the small to 
medium-sized enterprise: a divergent/convergent paradox in thinking patterns between advisers and SME owner-managers, Management Decision, 44(5), 581-597.

4 Voir à ce sujet Sadler-Smith, E. Hampson, Y., Chaston, I et Badger, B. (2003). Managerial behaviour; Entrepreneurial style, and small firm performance, Journal of Small Business Management, 41(1), 47-67; Smith, N.-R. et Miner, J.-B (1983). Type of Entrepreneur, type of firm, and managerial motivation: Implications for organizational life cycle theory, Strategic Management Journal, 4, 325-340.

5 Commission Européenne (2006). Communication de la commission au conseil, au parlement européen, au comité économique et social et au comité des régions. Bruxelles, 14 mars. www.ceder-reprendre-une-entreprise.com

6 Fédération canadienne des entreprises indépendantes (2005). La relève : la clé de la réussite, document de recherche, Fédération canadienne de l'entreprise indépendante (FCEI) http://www.cfib.ca/researchf/reports/rr3007f.pdf

7 Voir Mandl, I. (2004). Business transfers and successions in Austria, Austrian Institute for SME research. http://www.kmuforschung.ac.at/de/Forschungsberichte/Vortr\%C3\%A4ge/Business\%20Transfers\%20and\%20Successions\%20 in\%20Austria.pdf

8 APCE (2003). La reprise et la transmission d'entreprises. www.apce.com/upload/fichiers/observatoires/etudes/INFOPtransmission2003.pdf.

9 Cadieux, L. et Brouard, F. (2008). La transmission des PME : Perspectives et enjeux, Les Presses de l'Université du Québec, Sous presse.

10 APCE (2003). La reprise et la transmission d'entreprises. www.apce.com/upload/fichiers/observatoires/etudes/INFOPtransmission2003.pdf.; Transregio (2005). Enquête sur la transmission d'entreprise dans sept pays européens. www.transregio.org.

11 Ibid 7 .

12 Ibid 7. 


\section{Publicité}

\section{Site internet Revue OT}

